Trauma Berufskrankh 2004 - 6 [Suppl 2] : S262-\$265 DOI 10.1007/s10039-003-0769-1

Online publiziert: 26. August 2003

(c) Springer-Verlag 2003

M. Militz · V. Bühren • BG-Unfallklinik, Murnau

\title{
Sonderindikationen der winkelstabilen Plattenosteosynthese
}

Im oben genannten Zeitraum wurden

- 12 Pseudarthrosen (• Abb. 5),

- 6 Korrekturosteotomien,

- 4 Infektpseudarthrosen,

- 3 periprothetische Frakturen

(- Abb. 4) und

- 1 pathologische Fraktur (• Abb. 6)

mit winkelstabilen Implantaten versorgt.

Hinsichtlich der Lokalisation ist festzuhalten, dass in unserem Krankengut überwiegend Stabilisierungen an den oberen Extremitäten vorgenommen wurden, insbesondere bei Infekt- bzw. Pseudarthrosen.

\section{Diskussion}

Der Verwendung winkelstabiler Implantate zur Versorgung von Problemfrakturen bzw. Instabilitäten liegen die gleichen biomechanischen Prinzipien wie bei den Standardindikationen zugrunde. Durch die winkelstabile Verankerung der Schrauben in der Platte kann eine feste Verankerung des Implantats auch ohne direkten Knochenkontakt erzielt werden. Als vorteilhaft ist diesbezüglich anzusehen, dass die Platte bei den winkelstabilen Implantaten nicht durch das Einbringen der Schrauben an den Knochen angepresst werden muss (• Abb. 1). Dadurch ist eine geringere Beeinträchtigung der periostalen Durchblutung des bereits in vielen Fällen vorgeschädigten Knochens zu erwarten. Die winkelstabil verankerte Platte wirkt wie ein innerer Fixateur und kann aufgrund der festen Verankerung der Schrauben in der Platte auch monokortikal verankert werden.

Zur Implantation der winkelstabilen Implantate ist auch eine minimalinvasive Implantationstechnik geeignet (• Abb. 2).

Nachteilig wirkt sich aus, dass die Platten besonders an großen Röhrenknochen aufgrund der Dimensionierung schwer verformbar sind und die Schraubenlage aufgrund der winkelstabilen Verankerung in der Platte nicht wesentlich korrigierbar ist (• Abb. 3). Gelegentlich ist auch der bereits oben erwähnte Abstand zwischen der Platte und dem Knochen im Sinne eines inneren Fixateurs besonders bei geringer Weichteildeckung von Nachteil.

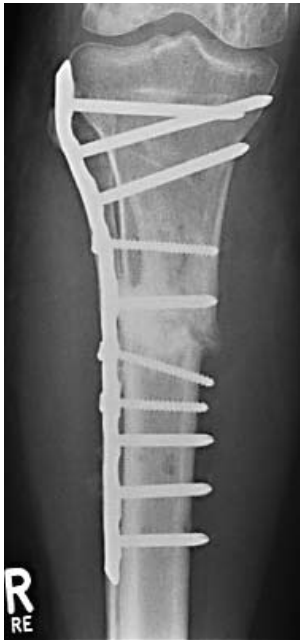

Abb. $1<$ Stabile Fixierung der Platte auch ohne Andruck an den Knochen, Verankerung der winkelstabilen Schrauben in der Platte vergleichbar einem inneren Fixateur 
Zur exakten Reposition insbesondere von Frakturen der langen Röhrenknochen ist die oben erwähnte Minimalinvasivität nicht immer durchzusetzen und dementsprechend ein offenes Zugehen erforderlich.

Bei

- Pseudarthrosen,

- periprothetischen Frakturen

(- Abb. 4),

- pathologischen Frakturen und

- Korrekturosteotomien

ist der Einsatz von winkelstabilen Implantaten aufgrund der oben angeführten Eigenschaften oft hilfreich. Insbesondere in Situationen, in denen eine Marknagelosteosynthese nicht möglich ist, bietet sich die winkelstabile Plattenosteosynthese an. Voraussetzung ist jedoch eine relative gute Weichteilsituation. Die winkelstabile Verankerung der Implantate lässt auch eine ausreichende stabile Situation für die postoperative Mobilisierung bei fraglicher Knochenqualität zu.

Bei Pseudarthrosen (• Abb. 5) ist in vielen Fällen aufgrund vorbestehender Behandlungsmaßnahmen und überschießender Knochenbildung bzw. gelenknaher Lokalisation die winkelstabile Plattenosteosynthese gegenüber dem Marknagel von Vorteil, da durch die zusätzliche Anlagerung von Spongiosa ein offener Zugang erforderlich ist, über den dann auch die Osteosynthese vorgenommen werden kann.

Ähnliches gilt für die Korrekturosteotomien. Hier ist insbesondere zu betonen dass aufgrund der winkelstabilen Verankerung der Schrauben im Implantat die korrigierte Stellung des Knochens nach unserer Erfahrung besser gehalten werden kann als in bisheriger Weise durch die konventionelle Plattenosteosynthese oder Marknagelung. Die stabile Verankerung der Schrauben im Implantat erlaubt eine frühzeitige funktionelle Therapie mit allen bekannten Vorteilen.

Eine weitere Indikation sind die periprothetischen Frakturen, wobei insbesondere am Oberschenkel durch Verlegung des Markraums bei einliegender Hüft-TEP eine Marknagelosteosynthese nicht in Frage kommen kann. Hier ist die stabile Verankerung der Platte mit monokortikal einge-

Trauma Berufskrankh 2004 - 6 [Suppl 2] : S262-S265

DOI 10.1007/s10039-003-0769-1

C Springer-Verlag 2003

\section{Militz · V. Bühren}

\section{Sonderindikationen der winkelstabilen Plattenosteosynthese}

\section{Zusammenfassung}

Die winkelstabile Plattenosteosynthese ist für die Versorgung von Problemfrakturen bzw. Instabilitäten geeignet, da die Vorteile der winkelstabilen Verankerung mit einer minimalinvasiven Implantationstechnik und postoperativer Funktionsstabilität erreicht werden können. Aus diesen Gründen ist die winkelstabile Plattenosteosynthese auch bei Problemversorgungen wie Pseudarthrosen, Infektpseudarthrosen, periprothetischen Frakturen, Korrekturosteotomien und pathologischen Frakturen indiziert. Nachteilig wirken sich die eingeschränkten Korrekturmöglichkeiten zur Applikation der Schrauben, das bei

großen Röhrenknochen relativ massive Implantat mit daraus resultierender Einschränkung der Modellierfähigkeit und der eingeschränkte Einsatz bei kritischer Weichteilsituation aus. Die Besonderheiten der Implantateigenschaften erfordern eine kritische Indikationsstellung und einen erfahrenen Operateur.

\section{Schlüsselwörter}

Winkelstabile Plattenosteosynthese Periprothetische Fraktur · Pseudarthrosen

Korrekturosteotomie

\section{Special indications for internal fixation with locked screw plates}

\section{Abstract}

Treatment with locked screw plates is suitable for problematic or unstable fractures, because the advantages of fixed-angle fixation can be reached with a minimally invasive implantation technique and functional stability after surgery. For these reasons, the locked screw plate is indicated even in such problematic situations as nonunion, periprosthetic fractures, revision osteotomies and pathologic fractures. Disadvantages can result from the restricted possibilities for correcting the application of the screws, the relatively massive implant needed when long bones are treated, which limits the capacity for modeling, and the restrictions on its use when critical soft tissue damage is present. These special characteristics of the implant mean that the indications for its use must be strictly defined and it should be inserted only by experienced surgeons.

\section{Keywords}

Locked screw plate - Periprosthetic fracture . Osteotomy · Nonunion 


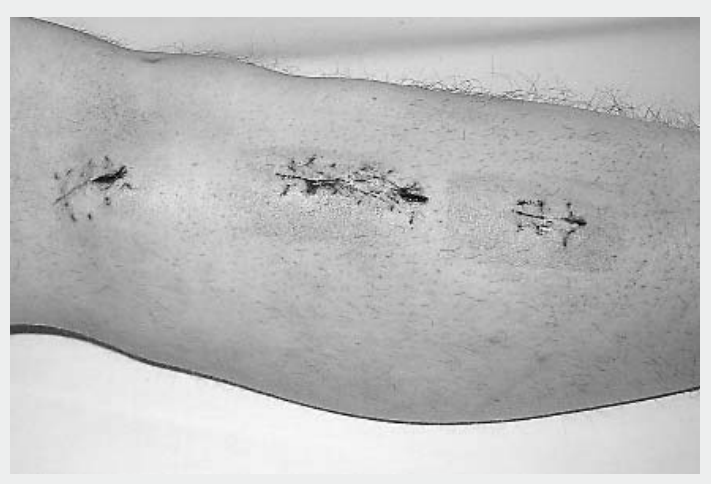

Abb. $2<$ Implantation der winkelstabilen Platte ist auch in minimalinvasiver Technik möglich
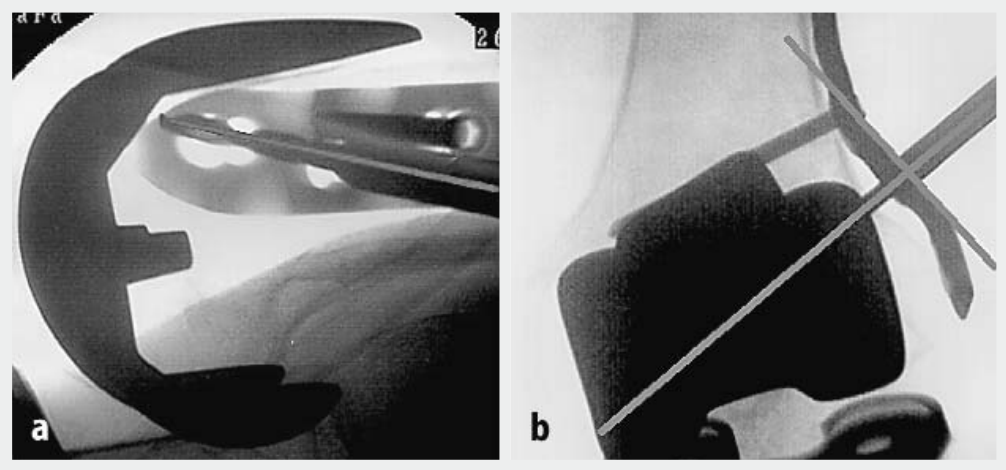

Abb. 3a,b $\Delta$ Lage der winkelstabilen Schrauben in der Platte wird durch das Verankerungsgewinde vorgegeben, hier Bohrung über die im Verankerungsgewinde eingeschraubte Bohrhülse
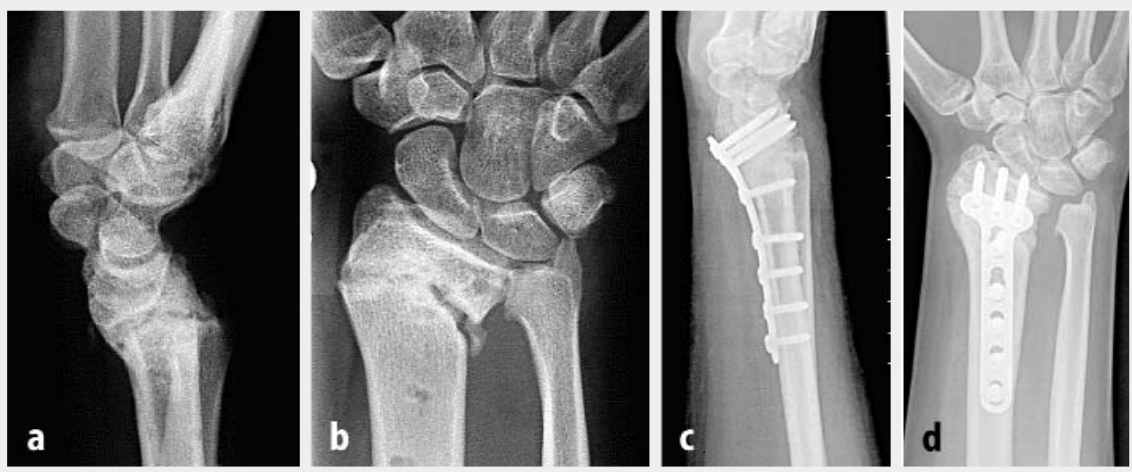

Abb. 5a-d $\Delta$ Distaler Radius, a,b Pseudarthrose mit Fehlstellung, c, d Konsolidierung nach Korrektur und Reosteosynthese mit winkelstabiler Platte von volar ohne Korrekturverlust

brachten Schrauben vorteilhaft (• Abb. 4). Außerdem erlaubt die winkelstabile Plattenosteosynthese eine frühzeitige Vollbelastung der betroffenen Extremität. Insbesondere bei periprothetischen Frakturen ist in vielen Fällen die Knochenqualität fraglich, sodass der winkelstabilen Verankerung des Implantats in diesen Fällen eine besondere Bedeutung zukommt.

Ähnliche Vorteile bietet die winkelstabile Plattenosteosynthese bei pathologischen Frakturen (- Abb. 6):
- Zur Gewinnung von Gewebeproben zur histologischen Untersuchung ist ein offenes Zugehen erforderlich.

- Bei pathologischen Frakturen ist die Marknagelosteosynthese aufgrund der Sklerosierung des Markraums nicht möglich.

- Die winkelstabile Plattenosteosynthese ist besonders zur Realisierung einer Verbundosteosynthese geeignet und gewährleistet eine stabile Verankerung, die auch für die postoperati-

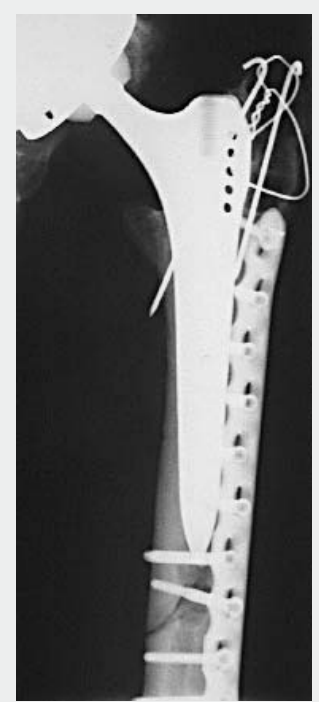

Abb. $4<$ Monokortikale Verankerung bei periprothetischer Fraktur mit massivem Hüft-TEP-Stiel
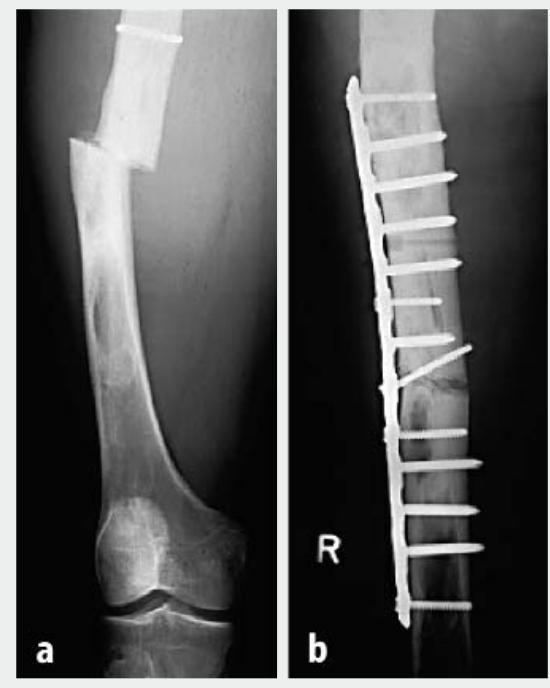

Abb. 6a,b $\Delta$ Pathologische Fraktur bei einem 25-jährigen Mann mit Osteofibrose, Versorgung mit winkelstabiler Platte

ve Mobilisierung des Patienten vorteilhaft ist.

\section{Korrespondierender Autor}

\section{Dr. M. Militz}

BG-Unfallklinik,

Prof.-Küntscher-Straße 8,82418 Murnau,

E-mail:militz@bgu-murnau.de 


\section{Literatur}

1. Beals RK, Stephen ST (1996) Periprosthetic fractures of the femur.Clin Orthop 327: 238-246

2. Berkhoff M, Meenen NM, Katzer A, Jungbluth K-H (1995) Erfahrungen mit der Plattenosteosynthese bei der Behandlung peri- und subprothetischer Femurfrakturen. Unfallchirurgie 21: 233-239

3. Bethea JSI, DeAnndrade JR, Fleming LL (1982) Proximal femoral fractures following total hip arthroplasty. Clin Orthop 170: 95

4. Blatter G, Fiechter Th, Magerl F (1989) Periprothetische Frakturen bei Hüfttotalendoprothesen. Orthopäde 18: 545-551

5. Buchholz J, Neumann K, Knopp W, Möllenhoff G, Muhr G (1995) Die hüftgelenknahe Femurfraktur bei einliegender Totalendoprothese. Chirurg 66: 1120-1125

6. Fabian W (1992) Gleichseitige Femurfraktur nach künstlichem Hüftgelenkersatz. Zentralbl Chir 117: 139-142

7. Faensen M (2000) Die Versorgung von periprothetischen Femurfrakturen bei liegender Kniegelenkprothese mit dem LIS-System. Unfallchirurg 103: 172

8. Hockertz TJ, Gruner A, Reilmann H (1999) Die Versorgung von periprothetischen Femurfrakturen bei liegender Kniegelenkprothese mit dem LIS-System. Unfallchirurg 102: 811-814

9. Johansson JE, McBroom R, Barrington TW, Hunter GA (1981) Fractures of the ipsilateral femur in patients with total hip arthroplasty. J Bone Joint Surg Am 63: 1435-1442

10. Kassab SS, Mast JW, Mayo KA (1998) Patients treated for nonunions with plate and screw fixation and adjunctive locking nuts. Clin Orthop 347: 86-92

11. Lonner JH, Siliski JM, Jupiter JB, Lhowe DW (1999) Posttraumatic nonunion of the proximal tibial metaphysis. Am J Orthop 28: 523-528

12. Neubauer Th, Wahler G, Kohlmann J, Osarovsky M, Wagner M (2001) Periprothetische Femurfrakturen bei liegendem Hüftimplantat. Eur JTrauma [Suppl 1]: 194-198

13. Palmer SH, Handley R, Willett $\mathrm{K}$ (2000) The use of interlocked 'customised' blade plates in the treatment of metaphyseal fractures in patients with poor bone stock. Injury 31: 187-191

14. Perren SM (2002) Evolution of the internal fixation of long bone fractures.J Bone Joint Surg Br 84: 10931110

15. Siegmeth $A$, Menth-Chiari W, Wozasek GE,Vécsei $V$ (1998) Die periprothetische Femurschaftfraktur: Indikation und Ergebnisse bei 51 Patienten. Unfallchirurg 101: 901-906

16. Zuber K, Koch P, Lustenberger A, Ganz R (1990) Femurfraktur nach Hüfttotalendoprothese. Unfallchirurg 93: $467-472$ 